\title{
Benefit and Risk of Prolonged Dual Antiplatelet Therapy After Percutaneous Coronary Intervention With Drug-Eluting Stents in Patients With Elevated Lipoprotein(a) Concentrations
}

OPEN ACCESS

Edited by:

Nathalie Pamir,

Oregon Health and Science University,

United States

Reviewed by:

Yuling Zhang

Sun Yat-sen Memorial Hospital, China

Emanuele Monda,

University of Campania Luigi

Vanvitelli, Italy

*Correspondence:

Lei Feng

fenglei0721@163.com

Kefei Dou

drdoukefei@126.com

tThese authors have contributed equally to this work

Specialty section:

This article was submitted to Lipids in Cardiovascular Disease,

a section of the journal

Frontiers in Cardiovascular Medicine

Received: 02 November 2021 Accepted: 29 November 2021

Published: 20 December 2021

Citation:

Cui $K$, Wang $H-Y$, Yin D, Zhu C, Song $W$, Wang $H$, Jia $L$, Zhang $D$, Song $C$, Feng $L$ and Dou $K$ (2021) Benefit and Risk of Prolonged Dual Antiplatelet Therapy After Percutaneous Coronary Intervention With Drug-Eluting Stents in Patients With Elevated Lipoprotein(a) Concentrations.

Front. Cardiovasc. Med. 8:807925. doi: 10.3389/fcvm.2021.807925

\author{
Kongyong Cui ${ }^{1,2,3,4+}$, Hao-Yu Wang ${ }^{1,2,3,4 t}$, Dong Yin ${ }^{1,2,3,4}$, Chenggang Zhu ${ }^{1,2,3,4}$, \\ Weihua Song ${ }^{1,2,3,4}$, Hongjian Wang ${ }^{1,2,3,4}$, Lei Jia ${ }^{1,2,3,4}$, Dong Zhang ${ }^{1,2,3,4}$, Chenxi Song ${ }^{1,2,3,4}$, \\ Lei Feng ${ }^{1,2,3,4 *}$ and Kefei Dou ${ }^{1,2,3,4 *}$ \\ ${ }^{1}$ Cardiometabolic Medicine Center, Department of Cardiology, Fuwai Hospital, National Center for Cardiovascular Diseases, \\ Chinese Academy of Medical Sciences and Peking Union Medical College, Beijing, China, ${ }^{2}$ Coronary Heart Disease Center, \\ Department of Cardiology, Fuwai Hospital, National Center for Cardiovascular Diseases, Chinese Academy of Medical \\ Sciences and Peking Union Medical College, Beijing, China, ${ }^{3}$ State Key Laboratory of Cardiovascular Disease, Beijing, \\ China, ${ }^{4}$ National Clinical Research Center for Cardiovascular Diseases, Beijing, China
}

Background: Lipoprotein(a) is positively related to cardiovascular events in patients with coronary artery disease (CAD). Given that lipoprotein(a) has a prothrombotic effect, prolonged dual antiplatelet therapy (DAPT) might have a beneficial effect on reducing ischemic events in patients with elevated lipoprotein(a) levels after percutaneous coronary intervention (PCl). We performed this study to assess the efficacy and safety of prolonged DAPT ( $>1$ year) in this population.

Methods: We evaluated a total of 3,025 CAD patients with elevated lipoprotein(a) levels who were event-free at 1 year after PCI from the prospective Fuwai PCI Registry, of which 913 received DAPT $\leq 1$ year and 2,112 received DAPT $>1$ year. The primary endpoint was major adverse cardiovascular and cerebrovascular event (MACCE), defined as a composite of all-cause death, myocardial infarction or stroke.

Results: After a median follow-up of 2.4 years, patients who received DAPT $>1$ year were associated with lower risks of MACCE compared with DAPT $\leq 1$ year (1.6 vs. 3.8\%; hazard ratio [HR] 0.383, 95\% confidence interval [Cl] 0.238-0.616), which was primarily driven by the lower all-cause mortality (0.2 vs. 2.3\%; HR 0.078, 95\% Cl $0.027-0.227$ ). In addition, DAPT>1 year was also associated with lower risks of cardiac death, and definite/probable stent thrombosis than those who received DAPT $\leq 1$ year $(P<0.05)$. Conversely, no difference was found between the two groups in terms of clinically relevant bleeding. Similar results were observed in multivariate Cox regression analysis and inverse probability of treatment weighting analysis.

Conclusions: In patients with elevated lipoprotein(a) concentrations after $\mathrm{PCl}$, prolonged DAPT (>1 year) reduced ischemic cardiovascular events, including MACCE, all-cause mortality, cardiac mortality, and definite/probable stent thrombosis, without 
increase in clinically relevant bleeding risk compared with $\leq 1$-year DAPT. Lipoprotein(a) levels might be a new important consideration when deciding the duration of DAPT after $\mathrm{PCl}$.

Keywords: lipoprotein(a) [Lp(a)], coronary artery disease, percutaneous coronary intervention (or PCl), drug-eluting stent (DES), DAPT (dual antiplatelet therapy), clinical outcome

\section{INTRODUCTION}

Lipoprotein(a) $[\mathrm{Lp}(\mathrm{a})]$ is a low-density lipoprotein (LDL)-like particle in which an apolipoprotein(a) [apo(a)] covalently linked to its apolipoprotein B100 component via a disulfide bridge (14). During the past decades, plasma Lp(a) has been recognized as a novel risk factor for the incidence of cardiovascular disease (5-11). Furthermore, an increasing evidence has supported that $\mathrm{Lp}$ (a) levels play an important role in predicting subsequent ischemic events in patients with established coronary artery disease (CAD), especially those who underwent percutaneous coronary intervention (PCI) (12-16). In a multicenter and prospective study, Liu et al. demonstrated that high $\mathrm{Lp}(\mathrm{a})$ levels were positively associated with adverse cardiovascular events in 4,078 stable CAD patients after PCI at a mean follow-up of 4.9 years (15). Nevertheless, there are still no approved pharmacologic therapies that specifically target high $\mathrm{Lp}$ (a) levels. Recently, as a novel therapeutic agent, hepatocytedirected antisense oligonucleotide APO(a)-LRx has been proven to reduce $L p(a)$ levels by $80 \%$ (17). However, the effect of this $\mathrm{Lp}(\mathrm{a})$-lowering drug on adverse cardiovascular events remains unknown.

Dual antiplatelet therapy (DAPT) consisting of aspirin and a $\mathrm{P}_{2} \mathrm{Y}_{12}$ receptor inhibitor represents the cornerstone of pharmacological treatment aimed at preventing thrombotic complications after PCI. Considering that $\mathrm{Lp}(\mathrm{a})$ has a prothrombotic effect through its inactive, plasminogen-like protease domain on apo(a) $(2,4)$, we speculate that prolonged DAPT may have a beneficial effect on reducing future ischemic events in patients who had elevated $\operatorname{Lp}(\mathrm{a})$ levels after PCI. However, the relative benefit of prolonged DAPT in this high-risk population has never been assessed. We therefore performed this study to compare the outcomes of prolonged DAPT ( $>1$ year) vs. shortened DAPT ( $\leq 1$ year) in patients with elevated $\operatorname{Lp}(\mathrm{a})$ levels who were event-free at 1 year after PCI with drug-eluting stent (DES) in a large and contemporary PCI registry.

\section{MATERIALS AND METHODS}

\section{Study Design and Population}

This was a retrospective analysis of a single-center, prospective registry and the study design has been previously described (18-20). Patients with CAD and elevated Lp(a) levels who underwent PCI with DES at Fuwai Hospital, National Center for Cardiovascular Diseases between January 2013 through December 2013 were consecutively enrolled. The study was performed according to the ethical guidelines of the 1975 Declaration of Helsinki and the study protocol has been priorly approved by the ethical committee of Fuwai Hospital, National Center for Cardiovascular Diseases. All the patients provided written informed consent before enrollment. Previous metaanalyses and the current guidelines for the management of dyslipidemia from China and Canada suggested that the relation between $\operatorname{Lp}(\mathrm{a})$ and cardiovascular risk inflects at a concentration of $30 \mathrm{mg} / \mathrm{dl}(8,14,21,22)$. We therefore used a cut-off of $>30$ $\mathrm{mg} / \mathrm{dl}$ to assign abnormal Lp(a) levels. For the present analysis, patients who had missing $\mathrm{Lp}(\mathrm{a})$ data $(n=665)$ or normal Lp(a) concentrations $(n=6,564)$, had follow-up duration $\leq 1$ year $(n$ $=35$ ) were not included. We also excluded patients who did not use DES $(n=137)$ or experienced adverse cardiovascular events (death, myocardial infarction [MI], stent thrombosis[ST], stroke, repeat revascularization, or Bleeding Academic Research Consortium [BARC] type 2, 3, or 5 bleeding) within 1-year follow-up $(n=298)$. Finally, 3,025 patients who met the selection criteria were divided into 2 groups according to the DAPT (aspirin plus clopidogrel) duration, i.e., DAPT $>1$-year group and DAPT $\leq 1$-year group. Landmark analysis was conducted to classify patients into treatment groups based on 1-year antiplatelet treatment after PCI and to evaluate prognosis from the landmark time point.

\section{Study Procedures and Biochemical Analysis}

All the procedures and medical therapies were performed in compliance with guidelines' recommendation and operators' discretion. Detailed information on procedures has been previously described $(19,20)$. After an overnight fasting, venous blood samples for measurement of $L p(a)$ and other biomarkers were obtained from all patients, and the test was conducted through clinical chemistry department in central lab of our hospital. Lp(a) was measured by immunoturbidimetry method [LASAY Lp(a) auto; SHIMA Laboratories Co., Ltd, Tokyo, Japan] with a normal value of $<30 \mathrm{mg} / \mathrm{dl}$. Levels of low-density lipoprotein cholesterol (LDL-C), high-density lipoprotein cholesterol, and total cholesterol were analyzed using the automated biochemical analyzer (Hitachi 7150, Tokyo, Japan), and glycosylated hemoglobin was tested with the Tosoh Automated Glycohemoglobin Analyser (HLC-723G8; Tosoh Corporation, Tokyo, Japan).

\section{Follow-Up and Endpoints}

Demographics, cardiovascular risk factors, clinical parameters, laboratory data, angiographic, and procedural details were prospectively collected in our dedicated PCI registry by independent research personnel. After index PCI, patients were followed up at 1, 6, and 12 months and annually thereafter. Data for endpoints were collected from medical records, clinical 
TABLE 1 | Baseline patient, angiographic, and procedural characteristics according to DAPT time.

\begin{tabular}{|c|c|c|c|}
\hline Variable & $\begin{array}{c}\text { DAPT } \leq 1 \\
\text { year }(n= \\
913)\end{array}$ & $\begin{array}{c}\text { DAPT > } 1 \\
\text { year }(n= \\
2,112)\end{array}$ & $P$-value \\
\hline Age, years & $58.6 \pm 10.1$ & $58.8 \pm 10.0$ & 0.850 \\
\hline Male, $n(\%)$ & $689(75.5)$ & $1,542(73.0)$ & 0.159 \\
\hline Body mass index, $\mathrm{kg} / \mathrm{m}^{2}$ & $25.6 \pm 3.1$ & $25.8 \pm 3.2$ & 0.409 \\
\hline Current smoker, $n(\%)$ & $515(56.4)$ & $1,120(53.0)$ & 0.087 \\
\hline Diabetes mellitus, $n$ (\%) & $266(29.1)$ & $603(28.6)$ & 0.745 \\
\hline Hypertension, $n(\%)$ & $579(63.4)$ & $1,384(65.5)$ & 0.264 \\
\hline Dyslipidemia, $n(\%)$ & $584(64.0)$ & $1,450(68.7)$ & 0.012 \\
\hline Previous myocardial infarction, $n(\%)$ & $161(17.6)$ & $419(19.8)$ & 0.157 \\
\hline Previous PCl, $n(\%)$ & $214(23.4)$ & $495(23.4)$ & 0.999 \\
\hline Previous CABG, $n(\%)$ & $39(4.3)$ & $103(4.9)$ & 0.470 \\
\hline Previous stroke, $n$ (\%) & $102(11.2)$ & $231(10.9)$ & 0.850 \\
\hline Peripheral vascular disease, $n(\%)$ & $19(2.1)$ & $59(2.8)$ & 0.256 \\
\hline Chronic kidney disease, $n(\%)$ & $86(9.4)$ & 233(11.0) & 0.185 \\
\hline COPD, $n(\%)$ & $30(3.3)$ & $55(2.6)$ & 0.298 \\
\hline LVEF, \% & $63.0 \pm 7.5$ & $62.9 \pm 7.3$ & 0.691 \\
\hline LVEF < $50 \%, n(\%)$ & $45(5.1)$ & $107(5.2)$ & 0.869 \\
\hline Acute coronary syndrome, $n(\%)$ & $568(62.2)$ & $1,205(57.1)$ & 0.008 \\
\hline Systolic blood pressure, $\mathrm{mmHg}$ & $127.1 \pm 17.8$ & $126.5 \pm 17.1$ & 0.379 \\
\hline $\begin{array}{l}\text { Laboratory data } \\
\text { WBC, } 10^{3} / \mathrm{UL}\end{array}$ & $6.76 \pm 1.66$ & $6.71 \pm 1.64$ & 0.630 \\
\hline Hemoglobin, g/L & $142.5 \pm 15.3$ & $142.3 \pm 15.7$ & 0.733 \\
\hline Total cholesterol, mmol/L & $4.33 \pm 1.04$ & $4.36 \pm 1.09$ & 0.634 \\
\hline LDL-C, mmol/L & $2.65 \pm 0.90$ & $2.66 \pm 0.93$ & 0.882 \\
\hline HDL-C, mmol/L & $1.06 \pm 0.28$ & $1.06 \pm 0.28$ & 0.798 \\
\hline $\mathrm{HbA} 1 \mathrm{c}, \%$ & $6.58 \pm 1.28$ & $6.62 \pm 1.22$ & 0.123 \\
\hline $\mathrm{Lp}(\mathrm{a}), \mathrm{mg} / \mathrm{dL}$ & $60.9 \pm 24.9$ & $60.9 \pm 24.5$ & 0.873 \\
\hline Radial artery access, $n(\%)$ & $775(92.4)$ & $1,758(90.4)$ & 0.093 \\
\hline Multivessel disease, $n(\%)$ & $682(74.7)$ & $1,629(77.1)$ & 0.148 \\
\hline SYNTAX score & $12.5 \pm 7.9$ & $12.3 \pm 7.8$ & 0.593 \\
\hline SYNTAX score $>22, n(\%)$ & $104(11.8)$ & 249 (12.3) & 0.720 \\
\hline Total lesion length, mm & $38.9 \pm 25.1$ & $39.9 \pm 26.7$ & 0.534 \\
\hline $\begin{array}{l}\text { Target lesion morphology } \\
\text { Bifurcation lesion, } n(\%)\end{array}$ & $192(21.0)$ & $429(20.3)$ & 0.654 \\
\hline 2-stent technique, $n$ (\%) & $37(4.1)$ & $98(4.6)$ & 0.472 \\
\hline Chronic total occlusion, $n(\%)$ & $167(18.3)$ & $421(19.9)$ & 0.295 \\
\hline In-stent restenosis, $n$ (\%) & $39(4.3)$ & $96(4.5)$ & 0.738 \\
\hline Severe calcification, $n(\%)$ & $27(3.0)$ & $67(3.2)$ & 0.754 \\
\hline Angulation > 45 degrees, $n(\%)$ & $99(10.8)$ & $235(11.1)$ & 0.819 \\
\hline Type B2 or C lesion, $n$ (\%) & $708(77.5)$ & $1,655(78.4)$ & 0.619 \\
\hline No. vessels treated & $1.30 \pm 0.51$ & $1.28 \pm 0.50$ & 0.321 \\
\hline No. lesions treated & $1.45 \pm 0.67$ & $1.44 \pm 0.68$ & 0.485 \\
\hline No. lesions treated $\geq 3, n(\%)$ & $69(7.6)$ & $146(6.9)$ & 0.526 \\
\hline Drug-eluting stent number & $1.91 \pm 1.02$ & $1.96 \pm 1.06$ & 0.344 \\
\hline Drug-eluting stent number $\geq 3, n(\%)$ & $220(24.1)$ & $502(23.8)$ & 0.846 \\
\hline Type of drug-eluting stent & & & 0.531 \\
\hline PES/SES, $n(\%)$ & $412(45.1)$ & $927(43.9)$ & \\
\hline EES/ZES, $n(\%)$ & $501(54.9)$ & $1,185(56.1)$ & \\
\hline Minimum stent diameter, mm & $2.91 \pm 0.49$ & $2.90 \pm 0.49$ & 0.524 \\
\hline Total stent length, mm & $42.9 \pm 25.9$ & $43.7 \pm 27.2$ & 0.534 \\
\hline
\end{tabular}

(Continued)
TABLE 1 | Continued

\begin{tabular}{lccc}
\hline Variable & $\begin{array}{c}\text { DAPT } \leq \mathbf{1} \\
\text { year }(\boldsymbol{n}= \\
\mathbf{9 1 3})\end{array}$ & $\begin{array}{c}\text { DAPT }>\mathbf{1} \\
\text { year }(\boldsymbol{n}= \\
\mathbf{2 , 1 1 2})\end{array}$ & $P$-value \\
\hline DAPT score & $1.61 \pm 1.25$ & $1.56 \pm 1.23$ & 0.300 \\
$\quad$ DAPT score $\geq 2, n(\%)$ & $498(54.5)$ & $1,128(53.4)$ & 0.565 \\
Medications at discharge & $900(98.6)$ & $2,088(98.9)$ & 0.509 \\
$\quad$ Aspirin, $n(\%)$ & & & \\
Clopidogrel, $n(\%)$ & $901(98.7)$ & $2,074(98.2)$ & 0.337 \\
$\beta$-blockers, $n(\%)$ & $828(90.7)$ & $1,931(91.4)$ & 0.509 \\
$\quad$ Statins, $n(\%)$ & $879(96.3)$ &, $2031(96.2)$ & 0.883 \\
$\quad$ Calcium channel blockers, $n(\%)$ & $442(48.4)$ & $1,041(49.3)$ & 0.657 \\
DAPT time, days & $350 \pm 56$ & $666 \pm 166$ & $<0.001$
\end{tabular}

$C A B G$, coronary artery bypass grafting; COPD, chronic obstructive pulmonary disease: $D A P T$, dual antiplatelet therapy; EES, everolimus-eluting stent; HDL-C, high-density lipoprotein cholesterol; $L D L-C$, low-density lipoprotein cholesterol; $L V E F$, left ventricular ejection fraction; PES, paclitaxel-eluting stent; $\mathrm{PCl}$, percutaneous coronary intervention; SES, sirolimus-eluting stent; WBC, white blood cell; ZES, zotarolimus-eluting stent.

visit, and telephone interviews by trained investigators who were blind to the clinical data. To record $\geq 2$-year follow-up information, the follow-up period was extended to January 31, 2016 for the present study. Adherence to antiplatelet medication was routinely assessed at each time of follow-up, and the status of antiplatelet therapy was collected by dedicated questionnaires and the electronic prescribing system at our center.

The primary endpoint was major adverse cardiovascular and cerebrovascular event (MACCE), defined as a composite of all-cause death, non-fatal MI, or stroke. Secondary endpoints included the individual components of the primary endpoint, cardiac death, definite or probable ST, and BARC type 2, 3, or 5 bleeding. Deaths were classified as either cardiac or non-cardiac. All deaths were considered to be cardiac-related unless a noncardiac origin was documented. MI was defined according to the third universal definition of MI (23). Stoke was defined as new focal neurological deficit lasting $>24 \mathrm{~h}$, which confirmed by a neurologist based on imaging evidence. Definite or probable ST was adjudicated on the basis of the Academic Research Consortium criteria (24). Bleeding events were categorized on the basis of the BARC classifications (25). Moreover, all the events were carefully verified and adjudicated by independent clinicians.

\section{Statistical Analysis}

Continuous variables were expressed as mean \pm standard deviation and categorical variables were expressed as frequencies (percentages). Differences in various characteristics were compared using Student's t test, Wilcoxon's rank sum test, Pearson's chi-square test, and Fisher's exact test, when appropriate. Cumulative incidence of clinical events was estimated using Kaplan-Meier curves, and differences were assessed with log-rank test. Univariate and multivariate Cox regression analyses were performed to calculate hazard ratios (HRs) and 95\% confidence intervals (CIs). Variables in Table 1 with $P<0.05$ in univariate analysis or those that were clinically relevant were entered into the multivariable 


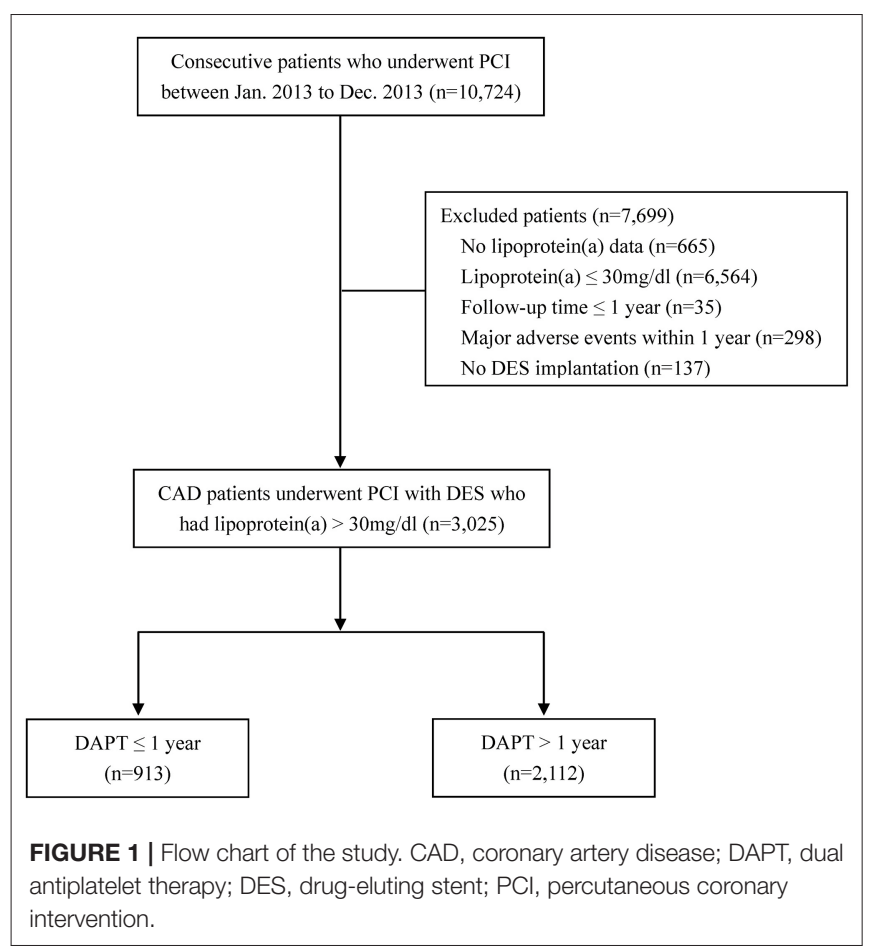

model, including age, sex, body mass index, current smoker, diabetes, hypertension, dyslipidemia, previous MI, previous PCI, previous coronary artery bypass graft surgery, previous stroke, peripheral vascular disease, acute coronary syndrome (ACS), total cholesterol, LDL-C, baseline SYNTAX score, multivessel disease, total lesion length, bifurcation lesion, in-stent restenosis, minimum stent diameter, total stent length, and use of statin at discharge.

Notably, inverse probability of treatment weighting (IPTW) analysis was also conducted to adjust for differences in baseline characteristics to evaluate the relative efficacy and safety of DAPT $>1$ year vs. DAPT $\leq 1$ year in patients with elevated Lp(a) levels. The propensity score was calculated using a non-parsimonious multivariable logistic regression model and considering DAPT time ( $>1$ year vs. $\leq 1$ year) as dependent variable. Covariates used for the propensity score model included age, sex, body mass index, current smoker, diabetes, hypertension, dyslipidemia, previous MI, previous PCI, previous stroke, peripheral vascular disease, chronic obstructive pulmonary disease, total cholesterol, LDL-C, total lesion length, type B2 or C lesion, chronic total occlusion (CTO), bifurcation lesion, number of lesions treated, stent number, use of everolimus- or zotarolimus-eluting stent, and use of aspirin, $\mathrm{P}_{2} \mathrm{Y}_{12}$ inhibitor, $\beta$-blocker, and statin at discharge. The detailed methods of IPTW analysis were previously described (26).

Furthermore, subgroup analysis of MACCE was performed based on the following factors, including age $(\leq 65$ and $>$ 65 years), sex, current smoking, diabetes, previous MI, chronic kidney disease (CKD), clinical presentation (stable angina vs. ACS), diseased vessels, type of DES, and DAPT score. All statistical analyses were conducted with SPSS version 23.0 (SPSS
Inc., Chicago, IL, USA) and R version 3.6.0 (R Foundation for Statistical Computing, Vienna, Austria). A two-sided $p<0.05$ was considered statistically significant.

\section{RESULTS}

\section{Baseline Characteristics}

Among the 3,025 patients with elevated Lp(a) concentrations $(>30 \mathrm{mg} / \mathrm{dl}$ ) who were event-free at 1 year after the index PCI, 913 received DAPT $\leq 1$ year and 2,112 received DAPT $>1$ year (Figure 1). The study participants had an average $\mathrm{Lp}(\mathrm{a})$ concentration of $60.9 \mathrm{mg} / \mathrm{dl}$, an average age of 58.7 years, and $73.8 \%$ of which were male. In addition, a large number of participants were considered to have traditional cardiovascular risk factors including hypertension (64.9\%), dyslipidemia (67.2\%), diabetes (28.7\%), and current smoking (54.0\%). Baseline patient, angiographic and procedural characteristics were similar between the two groups, except for the history of dyslipidemia and clinical presentation of acute coronary syndrome (Table 1). The median follow-up period was 2.4 $(2.2-2.6)$ years.

\section{Unadjusted Outcomes}

As shown in Table 2, Figures 2, 3, patients who received DAPT $>1$ year had lower risks of MACCE (1.6 vs. 3.8\%; HR 0.383, 95\% CI $0.238-0.616)$, which was primarily caused by the lower allcause mortality (0.2 vs. $2.3 \%$; HR 0.078 , 95\% CI $0.027-0.227)$. In addition, DAPT $>1$ year was also associated with lower risks of cardiac death (0.1 vs. $1.3 \%$; HR 0.103 , 95\%CI $0.029-0.366$ ), and definite/probable ST (0.4 vs. $1.3 \%$; HR 0.270 , 95\%CI $0.110-$ 0.662 ) than those who received DAPT $\leq 1$ year. Interestingly, no difference was found between the two groups in terms of BARC type 2, 3, or 5 bleeding (1.4 vs. $1.9 \%$; HR $0.720,95 \% \mathrm{CI}$ $0.397-1.307)$ at 2.4 years.

\section{Cox Proportional Hazards Regression Analysis}

After the potential confounders were adjusted, prolonged DAPT ( $>1$ year) remained associated with a reduced risk of MACCE ( $\mathrm{HR}_{\text {adjusted }} 0.335,95 \%$ CI $\left.0.202-0.555\right)$ at 2.4 years. Furthermore, prolonged DAPT was also a significant predictor of lower all-cause mortality (HR adjusted 0.056, 95\% CI 0.016-0.191), cardiac mortality (HR adjusted $0.056,95 \%$ CI $0.012-0.263$ ), and definite/probable ST (HR adjusted 0.173 , 95\% CI $0.063-0.474$ ) (Table 2).

\section{IPTW Analysis}

After IPTW adjustment, all the candidate variables were wellbalanced between DAPT $>1$-year and DAPT $\leq 1$-year groups, with absolute standardized differences $<10 \%$ (Figure 4). The IPTW analysis obtained consistent results that the risks of MACCE (HR IPTW 0.373, 95\% CI 0.231-0.601), all-cause death (HR IPTW 0.076, 95\%CI 0.026-0.223), cardiac death (HR IPTW $0.098,95 \%$ CI 0.027 to 0.352 ), and definite/probable ST (HR IPTW $_{0.253,95 \%}$ CI $\left.0.102-0.625\right)$ were significantly decreased in the prolonged DAPT group, and the risk of clinically relevant bleeding was not significantly different between the 
TABLE 2 | Clinical outcomes at 2.4 years according to DAPT time.

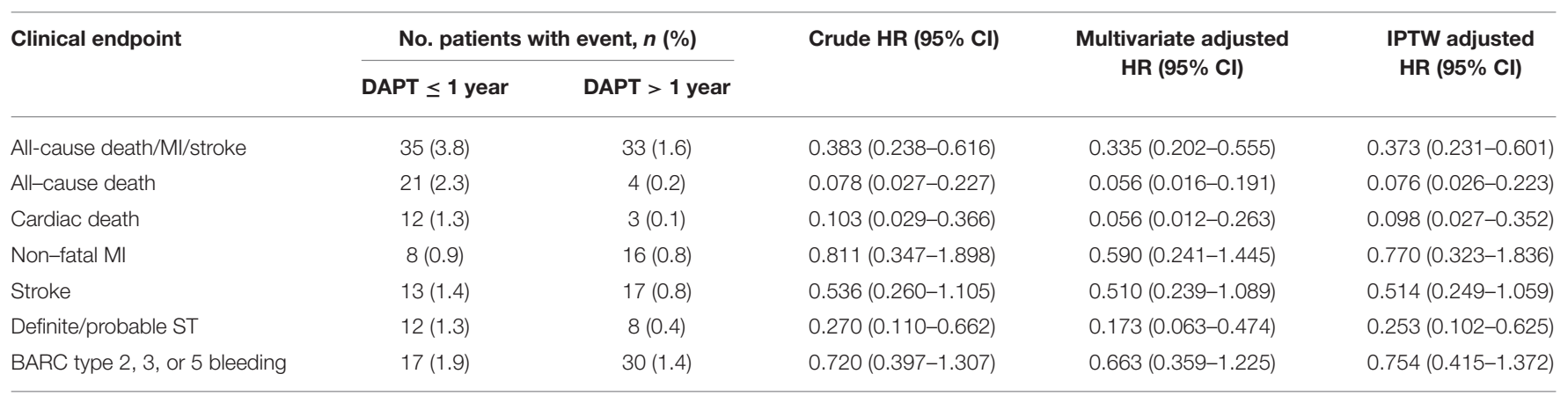

BARC, Bleeding Academic Research Consortium; Cl, confidence interval; DAPT, dual antiplatelet therapy; HR, hazard ratio; IPTW, inverse probability of treatment weighting; MI, myocardial infarction; ST, stent thrombosis.

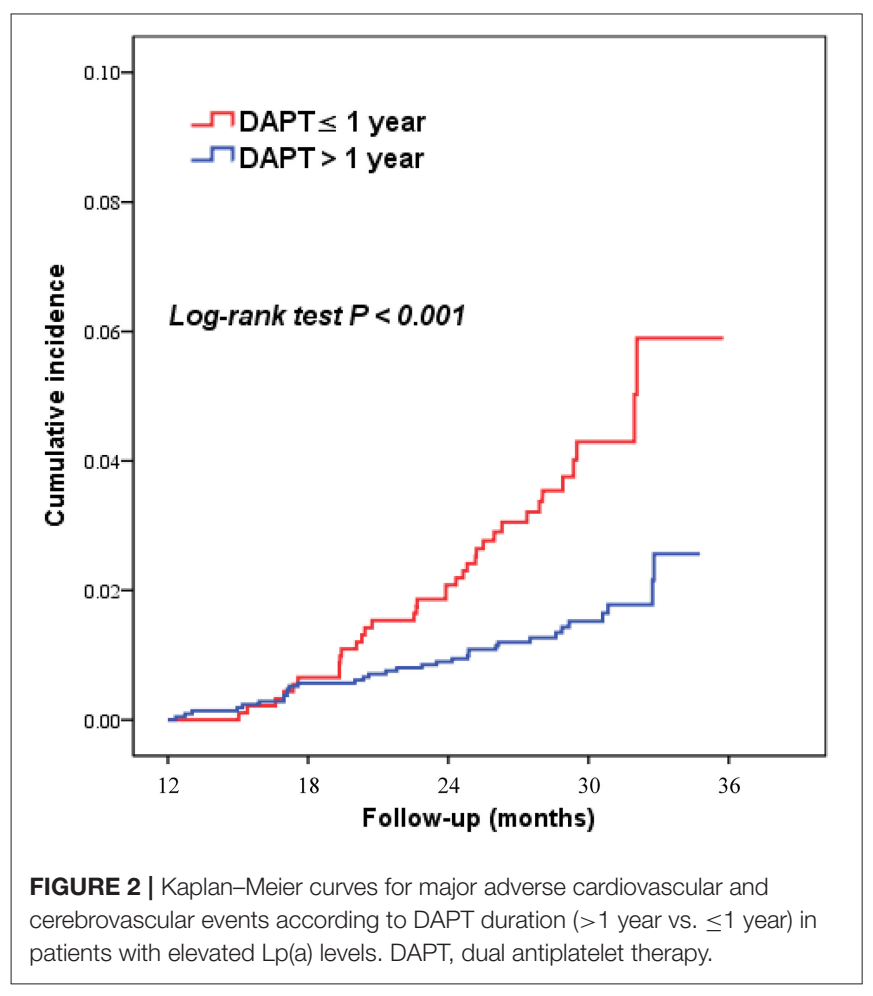

two antiplatelet therapies (HR IPTW $0.754,95 \%$ CI $0.415-1.372$ ) (Table 2).

\section{Subgroup Analysis}

Subgroup analyses were performed based on important baseline information, and formal testing for interactions indicated that results of the comparison of MACCE between the two groups were consistent across all the subgroups. The lower risk of MACCE in DAPT $>1$-year group than DAPT $\leq 1$-year group was consistently observed, regardless of age, gender, smoking status, diabetes, history of MI, CKD, clinical presentation, number of diseased vessels, type of DES, and DAPT score (Figure 5).

\section{DISCUSSION}

To the best our knowledge, this is the first study to evaluate the efficacy and safety of prolonged DAPT for CAD patients who had elevated Lp(a) concentrations after PCI with DES. We found that prolonged DAPT ( $>1$ year) was associated with lower risks of MACCE, all-cause mortality, cardiac mortality, and definite/probable ST, without increasing the risk of clinically relevant bleeding at 2.4 years. Furthermore, these favorable prognostic findings for DAPT $>1$-year against DAPT $\leq 1$ year were consistent across all the important clinical and procedural subgroups.

Plasma Lp(a) was initially described in 1963 by Berg K, and it has been recognized as a novel risk factor for cardiovascular disease in recent years. In 2008, Kamstrup et al. reported a stepwise increase in MI with increasing Lp(a) levels in 9,330 general participants from the Copenhagen City Heart Study (6). Henceforth, mounting evidence from meta-analyses, Mendelian randomization studies and genome-wide association studies indicated that $\mathrm{Lp}(\mathrm{a})$ was an independent, genetic, and causal risk factor for CAD (5, 7-11). Recently, some studies have indicated that $\mathrm{Lp}$ (a) levels was significantly associated with long-term adverse cardiovascular events in patients after PCI $(13,15)$. A study with 1,768 patients who received statin therapy after PCI showed that elevated $\mathrm{Lp}(\mathrm{a})$ levels were associated with increased cardiac death or ACS during a median follow-up of 4.4 years (HR adjusted 1.28, 95\%CI 1.04-1.58) (13). In addition, Liu et al. reported that high $\mathrm{Lp}(\mathrm{a})$ levels was associated with higher incidence of a composite of cardiac death, MI or stroke in stable CAD patients treated with statins after PCI at 4.9-year follow-up (15).

Nevertheless, there are still no approved pharmacologic therapies that specifically aimed to reduce $L p(a)$ levels. Evidence showed that the widely used statins have no $L p(a)$ lowering effect, and has even indicated a slight $\mathrm{Lp}(\mathrm{a})$ increasing effect. Though having a $20 \sim 30 \% \mathrm{Lp}(\mathrm{a})$-lowering effects, both niacin and mipomersen are associated with side effects, and mipomersen is only approved in homozygous familial hypercholesterolemia due to hepatotoxicity (2). PCSK9 inhibitor has been proved to reduce cardiovascular events independent of LDL-C in ACS patients by lowering $\mathrm{Lp}(\mathrm{a})$ levels, yet it could only predict a weak reduction in 

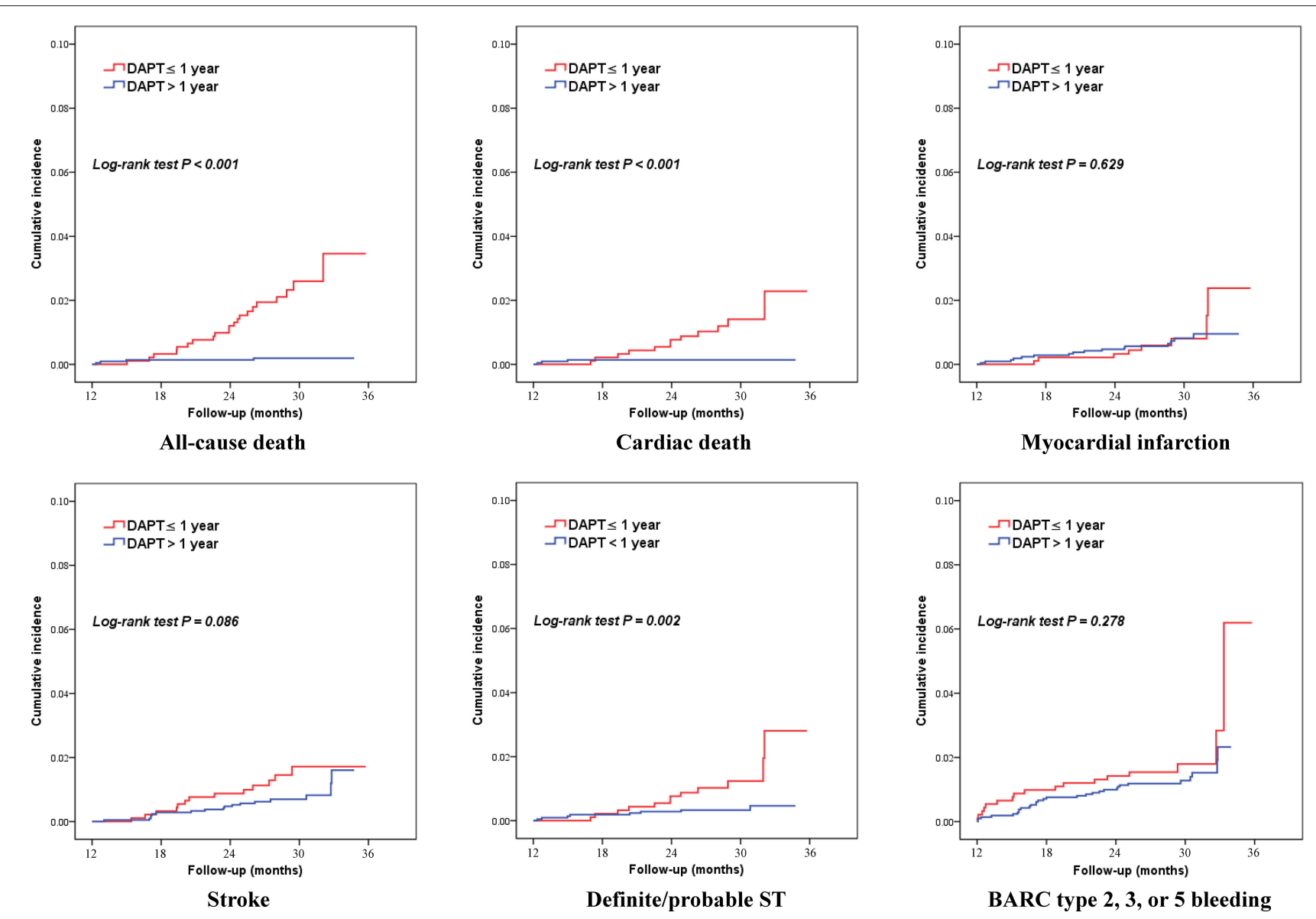

FIGURE 3 | Kaplan-Meier curves for secondary outcomes according to DAPT duration (>1 year vs. $\leq 1$ year) in patients with elevated Lp(a) levels. BARC, Bleeding Academic Research Consortium; DAPT, dual antiplatelet therapy; ST, stent thrombosis.

ischemic events (27). Actually, several Mendelian randomization analyses speculated that large absolute reduction in $\mathrm{Lp}(\mathrm{a})$ of $\sim 66-100 \mathrm{mg} / \mathrm{dl}$ may be required to achieve equivalent protective effects yielded from a $39-\mathrm{mg} / \mathrm{dL}$ (or $1-\mathrm{mmol} / \mathrm{L}$ ) reduction of LDL-C $(28,29)$. Tsimikas et al. found that a novel therapeutic agent, i.e., $\operatorname{APO}(\mathrm{a})-\mathrm{L}_{\mathrm{Rx}}$, provides potent reductions in levels of $\mathrm{Lp}$ (a) in patients with cardiovascular disease by reducing the production of apo(a) which offers greater specificity compared with PCSK9 inhibitor (17). Nonetheless, further trials are in demand to assess the impact of $L p(a)$ lowering with $A P O(a)-L_{R x}$ on major cardiac events in patients with established CAD.

Lp(a) consists of a LDL-like particle and an apo(a), which bounds to apolipoprotein B100 via a disulfide bond. It is generally believed that $\mathrm{Lp}$ (a) may contribute to cardiovascular disease by proatherogenic effects of the LDL-like component, proinflammatory effects of the oxidized phospholipid, and prothrombotic effects of the plasminogen-like apo(a) (1-4). For unknown etiological and physiological reasons, apo(a) has evolved from the plasminogen gene through duplication and remodeling. Due to the similarity between the apo(a) component of $\mathrm{Lp}(\mathrm{a})$ and plasminogen, $\mathrm{Lp}(\mathrm{a})$ promotes thrombotic and fibrinolytic events through several mechanisms, including inflammation through its content of oxidized phospholipids, the presence of lysine binding sites that allow accumulation in the arterial wall, and potential antifibrinolytic roles by inhibiting plasminogen activation $(2,4)$. Therefore, intensified antithrombotic therapy may have positive effect on patients with elevated Lp(a) levels after PCI. In this setting, it is reasonable that these high-risk patients could be benefited from extended DAPT by preventing thrombotic complications in long-term prognosis (30). Therefore, we compared the clinical outcomes of prolonged DAPT ( $>1$ year) vs. shortened DAPT $(\leq 1$ year) in patients with elevated Lp(a) levels who underwent PCI with DES.

Potentially the important finding of our study was that prolonged DAPT reduced the risks of MACCE, all-cause mortality, cardiac mortality, and definite or probable ST at 2.4 years in patients with elevated Lp(a) levels after PCI with DES. Additionally, the incidence of clinically relevant bleeding was similar between DAPT $>1$-year and DAPT $\leq 1$-year groups. Our previous study reported that DAPT continuation beyond 1 year offered a substantial reduction in ischemic cardiovascular events without apparent increase in clinically relevant bleeding risk 


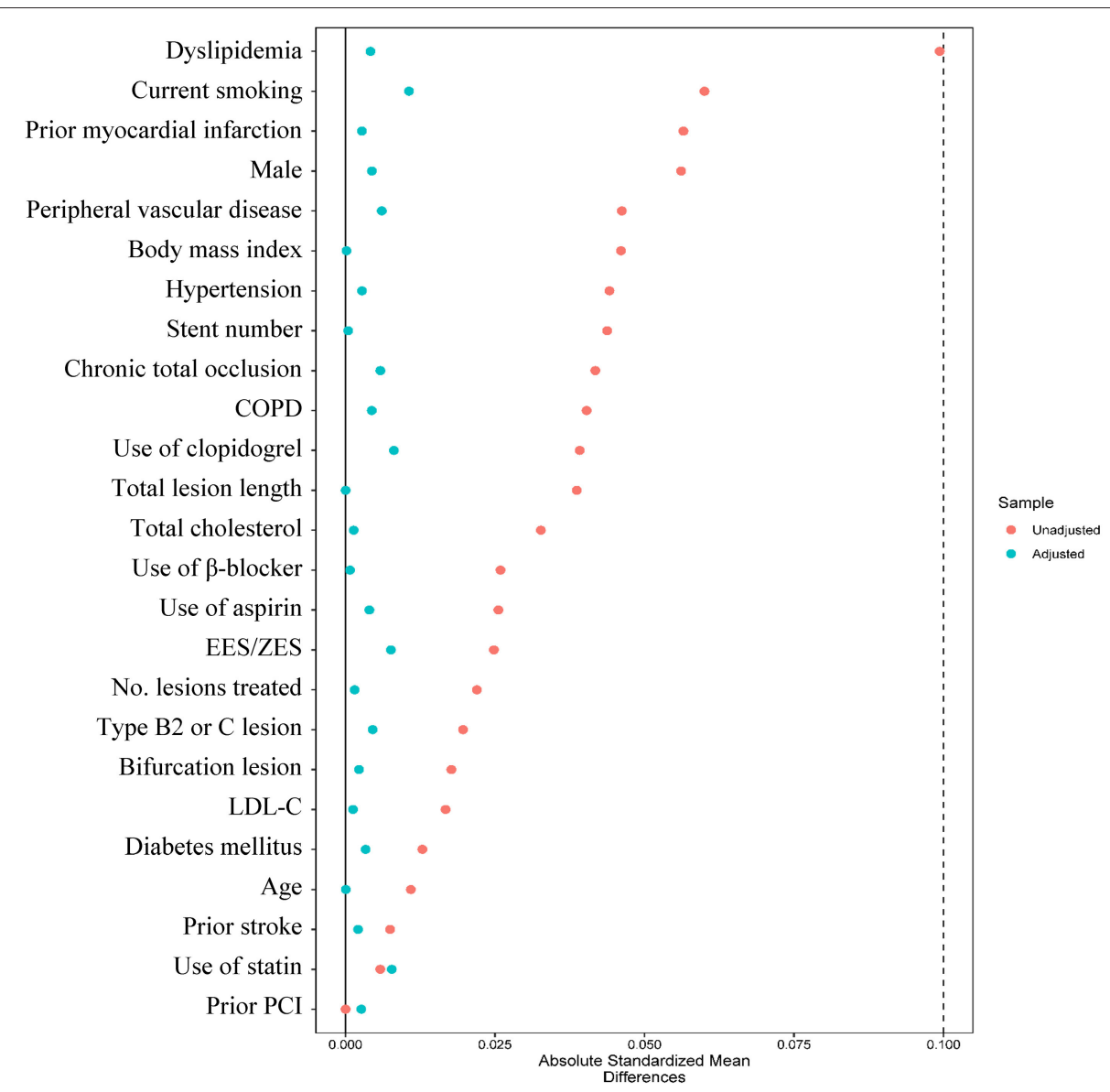

FIGURE 4 | Absolute standard difference before and after inverse probability of treatment weighting analysis between DAPT $>1$-year and DAPT $\leq 1$-year groups. COPD, chronic obstructive pulmonary disease; EES, everolimus-eluting stent; LDL-C, low-density lipoprotein cholesterol; PCI, percutaneous coronary intervention; ZES, zotarolimus-eluting stent.

compared with $\leq 1$-year DAPT in patients with ESC/EACTS guideline-endorsed HTR features (19). Of note, patients with CAD and elevated Lp(a) levels had been demonstrated to be at heightened risk for ischemic events, and considered as an important group with high ischemic risk. The current study found that these patients can benefit from prolonging DAPT duration after PCI with DES at a median follow-up of 2.4 years. In this setting, $\mathrm{Lp}(\mathrm{a})$ levels might be a new important consideration when deciding the duration of DAPT after PCI in the future.

However, our study presented several limitations. First, this is a single-center, non-randomized study, and it is limited by unbalanced baseline characteristics and selection bias. Actually, the duration of DAPT was not predefined but was individualized by physician discretion. Although rigorous multivariable-adjusted analysis and IPTW analysis were performed, it was hard to control all the confounding factors and eliminate the selection bias. Nonetheless, our findings reflect the real-world practice in that treatment was tailored to individual patient risk, as is recommended in current guidelines. Second, DAPT regimen in our study was based on the use of clopidogrel and aspirin; therefore, the clinical impact of DAPT $>1$ year with more potent $\mathrm{P}_{2} \mathrm{Y}_{12}$ inhibitor plus aspirin in CAD patients with high $\mathrm{Lp}(\mathrm{a})$ concentrations after PCI remains unclear. Third, $L p(a)$ was determined as mass concentration other than particle concentration, thus variations of apo(a) size between assay calibrators and patients' samples might overestimate or underestimate the real level of $\mathrm{Lp}(\mathrm{a})$. Luckily, a $\mathrm{Lp}(\mathrm{a})$ protein validated standard was used to calibrate the examination, along with linking the results to the World Health Organization/International Federation of Clinical Chemistry and Laboratory Medicine International Reference Reagent, making the assay relatively isoform independent. Fourth, the follow-up time should be extended to better specify the effect of prolonged DAPT on long-term outcomes.

\section{CONCLUSIONS}

In patients with elevated $\mathrm{Lp}$ (a) concentrations who were eventfree at 1 year after PCI with DES, prolonged DAPT ( $>1$ year) 


\begin{tabular}{|c|c|c|c|c|c|c|}
\hline Subgroup & \multirow{2}{*}{\multicolumn{2}{|c|}{ Event/Total (\%) }} & \multirow{2}{*}{\multicolumn{2}{|c|}{ Forest plot }} & \multirow[t]{2}{*}{$\begin{array}{c}\text { Hazard ratio } \\
(95 \% \mathrm{CI})\end{array}$} & \multirow{2}{*}{$\begin{array}{c}P \text { for } \\
\text { Interaction } \\
0.958\end{array}$} \\
\hline Age & & & & & & \\
\hline$\leqslant 65$ years & $19 / 681(2.8 \%)$ & 18/1572(1.1\%) & & 1 & $0.391(0.205-0.746)$ & \\
\hline$>65$ years & $16 / 232(6.9 \%)$ & $15 / 540(2.8 \%)$ & & 1 & $0.381(0.188-0.772)$ & \\
\hline Sex & & & & 1 & & 0.733 \\
\hline Male & $26 / 689(3.8 \%)$ & $25 / 1542(1.6 \%)$ & & 1 & $0.402(0.232-0.697)$ & \\
\hline Female & $9 / 224(4.0 \%)$ & $8 / 570(1.4 \%)$ & 一 & 1 & $0.332(0.128-0.861)$ & \\
\hline Current smoker & & & & 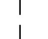 & & 0.670 \\
\hline Yes & $20 / 515(3.9 \%)$ & $16 / 1120(1.4 \%)$ & - & 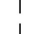 & $0.346(0.179-0.669)$ & \\
\hline No & $15 / 398(3.8 \%)$ & 17/992 (1.7\%) & & 1 & $0.426(0.213-0.854)$ & \\
\hline Diabetes mellitus & & & & 1 & & 0.896 \\
\hline Yes & $13 / 266(4.9 \%)$ & $12 / 603(2.0 \%)$ & & 1 & $0.366(0.167-0.805)$ & \\
\hline No & $22 / 647(3.4 \%)$ & $21 / 1509(1.4 \%)$ & & 1 & $0.391(0.215-0.711)$ & \\
\hline Previous myocardial infaretio & & & & I & & 0.952 \\
\hline Yes & $10 / 161(6.2 \%)$ & $10 / 419(2.4 \%)$ & & -1 & $0.368(0.153-0.885)$ & \\
\hline No & $25 / 752(3.3 \%)$ & $23 / 1693(1.4 \%)$ & - & I & $0.380(0.216-0.670)$ & \\
\hline Chronic kidney disease & & & & I & & 0.189 \\
\hline Yes & $3 / 86(3.5 \%)$ & $7 / 233(3.0 \%)$ & & + & $0.872(0.225-3.371)$ & \\
\hline No & $32 / 827(3.9 \%)$ & $26 / 1879(1.4 \%)$ & & I & $0.330(0.196-0.554)$ & \\
\hline Clinical presentation & & & & I & & 0.940 \\
\hline Stable angina & $15 / 345(4.3 \%)$ & $16 / 907(1.8 \%)$ & & i & $0.385(0.190-0.779)$ & \\
\hline Acute coronary syndrome & $20 / 568(3.5 \%)$ & $17 / 1205(1.4 \%)$ & & i & $0.371(0.194-0.709)$ & \\
\hline Diseased vessels & & & & 1 & & 0.296 \\
\hline Single-vessel disease & $5 / 231(2.2 \%)$ & 2/483 (0.4\%) & $\leftarrow$ & 1 & $0.165(0.032-0.853)$ & \\
\hline Multivessel disease & $30 / 682(4.4 \%)$ & $31 / 1629(1.9 \%)$ & - & 1 & $0.412(0.249-0.681)$ & \\
\hline Type of DES used & & & & 1 & & 0.165 \\
\hline PES/SES & $17 / 412(4.1 \%)$ & $10 / 927(1.1 \%)$ & -9 & 1 & $0.248(0.114-0.543)$ & \\
\hline EES/ZES & $18 / 501(3.6 \%)$ & $23 / 1185(1.9 \%)$ & & -1 & $0.502(0.270-0.931)$ & \\
\hline DAPT score & & & & 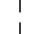 & & 0.346 \\
\hline$<2$ & $20 / 415(4.8 \%)$ & $15 / 984(1.5 \%)$ & 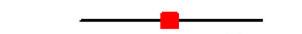 & 1 & $0.308(0.158-0.601)$ & \\
\hline$\geq 2$ & $15 / 498(3.0 \%)$ & $18 / 1128(1.6 \%)$ & & - & $0.488(0.246-0.971)$ & \\
\hline & & & $T$ & + & & \\
\hline & & & .1 & 1 & & \\
\hline
\end{tabular}

reduced ischemic cardiovascular events, including MACCE, allcause mortality, cardiac mortality, and definite/probable ST, without increase in clinically relevant bleeding risk compared with $\leq 1$-year DAPT. Further well-designed, randomized trials are needed to confirm these findings.

\section{DATA AVAILABILITY STATEMENT}

The raw data supporting the conclusions of this article will be made available by the authors, without undue reservation.

\section{ETHICS STATEMENT}

The studies involving human participants were reviewed and approved by the Ethical Committee of Fuwai Hospital, National Center for Cardiovascular Diseases. The patients/participants provided their written informed consent to participate in this study.

\section{REFERENCES}

1. Wilson DP, Jacobson TA, Jones PH, Koschinsky ML, McNeal CJ, Nordestgaard BG, et al. Use of Lipoprotein(a) in clinical practice: a biomarker whose time has come. a scientific statement from the National Lipid

\section{AUTHOR CONTRIBUTIONS}

$\mathrm{KC}, \mathrm{LF}$, and $\mathrm{KD}$ : study design and interpretation of results. DY, CZ, WS, HW, LJ, LF, and KD: angiography review and patient enrollment. DZ, CS, KC, and H-YW: data collection. KC, WS, HW, and LJ: data analysis. KC and H-YW: preparation of manuscript. KC, H-YW, LF, and KD: revision of manuscript. All authors contributed to the article and approved the submitted version.

\section{FUNDING}

This study was funded by CAMS Innovation Fund for Medical Sciences (CIFMS) (2021-I2M-1-008) and Beijing Municipal Health Commission-Capital Health Development Research Project (2020-1-4032). Sponsors were not involved in study design; in the collection, analysis, and interpretation of data; in the writing of the report; and in the decision to submit the article for publication.

Association. J Clin Lipidol. (2019) 13:374-92. doi: 10.1016/j.jacl.2019. 04.010

2. Tsimikas S. A test in context: Lipoprotein(a): diagnosis, prognosis, controversies, and emerging therapies. J Am Coll Cardiol. (2017) 69:692711. doi: 10.1016/j.jacc.2016.11.042 
3. Mach F, Baigent C, Catapano AL, Koskinas KC, Casula M, Badimon L, et al. 2019 ESC/EAS Guidelines for the management of dyslipidaemias: lipid modification to reduce cardiovascular risk. Eur Heart J. (2020) 41:11188. doi: 10.15829/1560-4071-2020-3826

4. Tsimikas S, Fazio S, Ferdinand KC, Ginsberg HN, Koschinsky ML, Marcovina SM, et al. NHLBI Working Group recommendations to reduce Lipoprotein(a)-mediated risk of cardiovascular disease and aortic stenosis. $J$ Am Coll Cardiol. (2018) 71:177-92. doi: 10.1016/j.jacc.2017.11.014

5. Danesh J, Collins R, Peto R. Lipoprotein(a) and coronary heart disease. meta-analysis of prospective studies. Circulation. (2000) 102:1082-5. doi: 10.1161/01.CIR.102.10.1082

6. Kamstrup PR, Benn M, Tybjaerg-Hansen A, Nordestgaard BG. Extreme lipoprotein(a) levels and risk of myocardial infarction in the general population: the Copenhagen City Heart Study. Circulation. (2008) 117:17684. doi: 10.1161/CIRCULATIONAHA.107.715698

7. Clarke R, Peden JF, Hopewell JC, Kyriakou T, Goel A, Heath SC, et al. Genetic variants associated with $\mathrm{Lp}$ (a) lipoprotein level and coronary disease. $\mathrm{N} \mathrm{Engl} \mathrm{J}$ Med. (2009) 361:2518-28. doi: 10.1056/NEJMoa0902604

8. Emerging Risk Factors Collaboration, Erqou S, Kaptoge S, Perry PL, Di Angelantonio E, Thompson A, et al. Lipoprotein(a) concentration and the risk of coronary heart disease, stroke, and nonvascular mortality. JAMA. (2009) 302:412-23. doi: 10.1001/jama.2009.1063

9. Kamstrup PR, Tybjaerg-Hansen A, Steffensen R, Nordestgaard BG. Genetically elevated lipoprotein(a) and increased risk of myocardial infarction. JAMA. (2009) 301:2331-9. doi: 10.1001/jama.2009.801

10. Wei WQ, Li X, Feng Q, Kubo M, Kullo IJ, Peissig PL, et al. LPA variants are associated with residual cardiovascular risk in patients receiving statins. Circulation. (2018) 138:183949. doi: 10.1161/CIRCULATIONAHA.117.031356

11. Langsted A, Kamstrup PR, Nordestgaard BG. High lipoprotein(a) and high risk of mortality. Eur Heart J. (2019) 40:276070. doi: 10.1093/eurheartj/ehy902

12. O’Donoghue ML, Morrow DA, Tsimikas S, Sloan S, Ren AF, Hoffman EB, et al. Lipoprotein(a) for risk assessment in patients with established coronary artery disease. J Am Coll Cardiol. (2014) 63:520-7. doi: 10.1016/j.jacc.2013. 09.042

13. Suwa S, Ogita M, Miyauchi K, Sonoda T, Konishi H, Tsuboi S, et al. Impact of Lipoprotein (a) on long-term outcomes in patients with coronary artery disease treated with statin after a first percutaneous coronary intervention. J Atheroscler Thromb. (2017) 24:1125-31. doi: 10.5551/jat. 38794

14. Willeit P, Ridker PM, Nestel PJ, Simes J, Tonkin AM, Pedersen TR, et al. Baseline and on-statin treatment lipoprotein(a) levels for prediction of cardiovascular events: individual patient-data meta-analysis of statin outcome trials. Lancet. (2018) 392:1311-20. doi: 10.1016/S0140-6736(18)31652-0

15. Liu HH, Cao YX, Jin JL, Zhang HW, Hua Q, Li YF, et al. Predicting cardiovascular outcomes by baseline Lipoprotein(a) concentrations: a large cohort and long-term follow-up study on real-world patients receiving percutaneous coronary intervention. J Am Heart Assoc. (2020) 9:e014581. doi: 10.1161/JAHA.119.014581

16. Shah NP, Wang Q, Wolski KE, Cho L, McErlean E, Ruotolo G, et al. The role of Lipoprotein (a) as a marker of residual risk in patients with diabetes and established cardiovascular disease on optimal medical therapy: post hoc analysis of ACCELERATE. Diabetes Care. (2020) 43:e224. doi: $10.2337 / \mathrm{dc} 19-1117$

17. Tsimikas S, Karwatowska-Prokopczuk E, Gouni-Berthold I, Tardif JC, Baum SJ, Steinhagen-Thiessen E, et al. Lipoprotein(a) reduction in persons with cardiovascular disease. N Engl J Med. (2020) 382:24455. doi: 10.1056/NEJMoa1905239

18. Zhang D, Yan R, Gao G, Wang H, Fu R, Li J, et al. Validating the performance of 5 risk scores for major adverse cardiac events in patients who achieved complete revascularization after percutaneous coronary intervention. Can J Cardiol. (2019) 35:1058-68. doi: 10.1016/j.cjca.2019.02.017

19. Wang HY, Dou KF, Wang Y, Yin D, Xu B, Gao RL. Benefit-risk profile of DAPT continuation beyond 1 year after PCI in patients with high thrombotic risk features as endorsed by 2018. ESC/EACTS myocardial revascularization guideline. Cardiovasc Drugs Ther. (2020) 34:663-75. doi: 10.1007/s10557-020-07030-9

20. Wang HY, Gao RL, Xu B, Yang YJ, Yin D, Wang Y, et al. Benefit-risk profile of extended dual antiplatelet therapy beyond 1 year in patients with high risk of ischemic or bleeding events after PCI. Platelets. (2021) 32:53341. doi: 10.1080/09537104.2020.1774052

21. Joint committee issued Chinese guideline for the management of dyslipidemia in a. [2016 Chinese guideline for the management of dyslipidemia in adults]. Zhonghua Xin Xue Guan Bing Za Zhi. (2016) 44:83353. doi: 10.3760/cma.j.issn.0253-3758.2016.10.005

22. Anderson TJ, Grégoire J, Pearson GJ, Barry AR, Couture P, Dawes M, et al. 2016 Canadian cardiovascular society guidelines for the management of dyslipidemia for the prevention of cardiovascular disease in the adult. Can J Cardiol. (2016) 32:1263-82. doi: 10.1016/j.cjca.2016.07.510

23. Thygesen K, Alpert JS, Jaffe AS, Simoons ML, Chaitman BR, White HD, et al. Third universal definition of myocardial infarction. Eur Heart J. (2012) 33:2551-67. doi: 10.1016/j.gheart.2012.08.001

24. Cutlip DE, Windecker S, Mehran R, Boam A, Cohen DJ, van Es GA, et al. Clinical end points in coronary stent trials: a case for standardized definitions. Circulation. (2007) 115:234451. doi: 10.1161/CIRCULATIONAHA.106.685313

25. Mehran R, Rao SV, Bhatt DL, Gibson CM, Caixeta A, Eikelboom J, et al. Standardized bleeding definitions for cardiovascular clinical trials: a consensus report from the Bleeding Academic Research Consortium. Circulation. (2011) 123:2736-47. doi: 10.1161/CIRCULATIONAHA.110.009449

26. Wang HY, Cai ZX, Yin D, Song WH, Feng L, Gao RL, et al. Optimal strategy for antiplatelet therapy after coronary drug-eluting stent implantation in highrisk "TWILIGHT-like" patients with diabetes mellitus. Front Cardiovasc Med. (2020) 7:586491. doi: 10.3389/fcvm.2020.586491

27. Szarek M, Bittner VA, Aylward P, Baccara-Dinet M, Bhatt DL, Diaz R, et al. Lipoprotein(a) lowering by alirocumab reduces the total burden of cardiovascular events independent of low-density lipoprotein cholesterol lowering: ODYSSEY OUTCOMES trial. Eur Heart J. (2020) 41:424555. doi: 10.1093/eurheartj/ehaa649

28. Burgess S, Ference BA, Staley JR, Freitag DF, Mason AM, Nielsen SF, et al. Association of LPA variants with risk of coronary disease and the implications for Lipoprotein(a)-lowering therapies: a mendelian randomization analysis. JAMA Cardiol. (2018) 3:619-27. doi: 10.1001/jamacardio.2018.1470

29. Lamina C, Kronenberg F; Lp(a)-GWAS-Consortium. Estimation of the required Lipoprotein(a)-lowering therapeutic effect size for reduction in coronary heart disease outcomes: a mendelian randomization analysis. JAMA Cardiol. (2019) 4:575-9. doi: 10.1001/jamacardio.2019.1041

30. Miksenas H, Januzzi JL Jr, Natarajan P. Lipoprotein(a) and cardiovascular diseases. JAMA. (2021) 326:352-3. doi: 10.1001/jama.2021.3632

Conflict of Interest: The authors declare that the research was conducted in the absence of any commercial or financial relationships that could be construed as a potential conflict of interest.

Publisher's Note: All claims expressed in this article are solely those of the authors and do not necessarily represent those of their affiliated organizations, or those of the publisher, the editors and the reviewers. Any product that may be evaluated in this article, or claim that may be made by its manufacturer, is not guaranteed or endorsed by the publisher.

Copyright (c) 2021 Cui, Wang, Yin, Zhu, Song, Wang, Jia, Zhang, Song, Feng and Dou. This is an open-access article distributed under the terms of the Creative Commons Attribution License (CC BY). The use, distribution or reproduction in other forums is permitted, provided the original author(s) and the copyright owner(s) are credited and that the original publication in this journal is cited, in accordance with accepted academic practice. No use, distribution or reproduction is permitted which does not comply with these terms. 